\title{
Exploring Binge Eating and Physical Activity among Community-Dwelling Women
}

\author{
K. Jason Crandall ${ }^{1, *}$, Patricia A. Eisenman ${ }^{2}$, Lynda Ransdell ${ }^{3}$, Justine Reel ${ }^{4}$ \\ ${ }^{1}$ Department of Kinesiology and Health Promotion, Kentucky Wesleyan College, 3000 Frederica Street F.O.B. 19 \\ ${ }^{2}$ College of Health, Department of Exercise and Sport Science, University of Utah \\ ${ }^{3}$ Department of Kinesiology, Boise State University \\ ${ }^{4}$ Department of Health Promotion and Education, University of Utah
}

\begin{abstract}
The primary aim was to understand physical activity (PA) changes throughout the life spans of binge eating disordered (BED) participants. A secondary aim was to determine if barriers exists that limit PA participation. METHODS: Females $(\mathrm{N}=312)$ were recruited within the continental United States and self-administered questionnaires. BED participants $(\mathrm{n}=18)$, subclinical BED participants $(\mathrm{n}=19)$, and non-BED overweight controls $(\mathrm{n}=19)$ were identified. Demographic variables, PA levels, and exercise perceived benefits and barriers scores were compared. RESULTS: BED individuals reported lower PA levels during the young adult and mature adult periods when compared to non-BED body weight matched controls. Significant differences were found between BED individuals and controls for six benefits and barriers questions ( $p$ $<.05)$. BED individuals have lower PA compared to controls during periods of the life span, and exercise benefits and barriers are significantly different. Specific barriers need to be addressed if PA is to be used as an adjunct treatment for BED individuals.
\end{abstract}

Keywords Binge Eating, Exercise, Physical Activity, Eating Disorders

\section{Introduction}

Binge Eating Disorder (BED) is an eating disorder characterized by episodes of binge eating without inappropriate compensatory behaviors such as excessive exercise, vomiting, or laxatives (Wonderlich, Gordon, Mirchell, Crosby, \& Engel, 2009). Prevalence rates for BED have been found to be as high as $40 \%$ in weight management samples and as low as $2 \%$ in the general population (Dingemans, Bruna, \& Furth, 2002). Individuals with BED tend to be overweight or obese due to binge eating episodes without the accompanying energy expenditure or purging methods (Brownley, Berkman, Sedway, Lohr, \& Bulik, 2007). Overweight or obese individuals who are diagnosed with BED demonstrate significantly more psychopathology and dietary disinhibition when compared to non-BED obese individuals (Grilo, White, \& Masheb, 2009). BED individuals also exhibit excessive concern with their body shape and lack of thinness, become overweight at a younger age, and spend more time on dieting efforts compared to their non-BED counterparts (Grilo et al., 2009). Due to these differences, obesity prevention approaches need to address the unique problems associated with obese BED individuals (Argas, 1995; Levine \& Marcus

\footnotetext{
* Corresponding author:

jcrandall@kwc.edu (K. Jason Crandall)

Published online at http://journal.sapub.org/ijpbs

Copyright () 2011 Scientific \& Academic Publishing. All Rights Reserved
}

2003). A key component of weight management programs, and a potential treatment for individuals with BED, is participation in structured or unstructured physical activity (PA) (Donnelley et al., 2009). Crandall and Eisenman (2001) asserted that PA has the potential to positively support the treatment of BED.

Historical PA patterns in individuals with BED are unknown. Researchers have shown that BED participants become overweight, begin dieting, and first meet the criteria for BED in early adolescence (Brownley et al., 2007). Overweight binge eating children also show greater levels of obesity and more psychopathology compared to overweight children who do not binge eat.

The current PA patterns of BED individuals who are not in treatment are unknown. There are no data are related to the type, frequency, duration, and overall level of PA. A review of the literature determined that overall energy expenditure, including activity-induced thermogenesis, in BED individuals has yet to be consistently identified (de Zwaan, Aslam, \& Mitchell, 2002). Although researchers have examined PA levels, energy expenditure, and perceived benefits and barriers to PA in individuals with anorexia nervosa and bulimia nervosa, this important information has yet to be elucidated in BED individuals (Davis et al, 1997). To date, two investigations have been conducted to determine the effects of structured PA on BED. Participants were instructed to walk between 3 and 5 days per week (expending $\sim 1,000 \mathrm{kcals} / \mathrm{wk}$ ) for 6 months (Levine, Marcus \&, Moulton, 1996). After a 6- 
month intervention, binge episodes were significantly decreased, however, body weight and depression did not change. Leisure time structured exercise was used in conjunction with cognitive behavior therapy (CBT) (Pendleton, Goodrick, Poston, Reeves, \& Foreyt, 2002). One hundred fourteen obese female binge eaters were randomized into four groups: (a) CBT with exercise and maintenance, (b) CBT with exercise, (c) CBT with maintenance, and (d) CBT only. The maintenance component consisted of 12 biweekly meetings over a period of 6 months after cessation of treatment. Eighty-four women completed the 16-month study. Participants in the exercise groups were expected to participate in a minimum of 45 minutes of cardiovascular exercise three times per week for 4 months. Two of the sessions each week were completed at an exercise facility, and the third session was completed at home. At the end of the study, the exercise groups had greater reductions in binge days compared with the non-exercise groups at 4 months, 10 months, and 16 months. The exercise groups attended an average of 16 out of a possible 32 exercise facility sessions. The exercise groups self-reported significantly more leisure time PA at the end of 4 months; however, when compared to the nonexercise groups at 10 and 16 months, no significant differences were found. Despite attending only half of the exercise facility sessions and reporting only slightly more PA levels than the nonexercise groups, the exercise groups significantly reduced binge eating episodes and experienced improvements in mood and reductions in body mass index as compared to the nonexercise groups. Possible improvements in physiological measures such as reduced body fat levels, blood glucose levels, blood pressure, and cholesterol were not monitored; therefore, it is unclear if the exercise training program affected risk factors for hypokinetic diseases. Other areas not addressed in the study included the limited quantification of PA, a lack of information about the relationships between formal PA instruction and binge eating status, and a lack of information about the low adherence to the exercise program. The results of these studies suggest that formal PA instruction may be an effective addition to BED treatment. However, structured physical activity interventions may be premature until more systematic research is conducted to better understand the role historical and current physical activity plays in the lives of BED individuals (Davis et al., 1997).

Historical PA patterns in BED participants are unknown. Researchers have shown that most BED participants become overweight, begin dieting, and first meet the criteria for BED in early adolescence (Brownley, et al., 2007). Overweight binge eating children also show greater levels of obesity and more psychopathology compared to overweight children who do not binge eat (Morgan et al., 2002).

The current PA patterns of BED individuals not in treatment are unknown. There are no data related to the type, frequency, duration, and overall level of PA. A review of the literature determined that overall energy expenditure, including activity-induced thermogenesis, in BED individuals has yet to be consistently identified. Although researchers have examined PA levels, energy expenditure, and perceived benefits and barriers to PA in individuals with anorexia nervosa and bulimia nervosa, this important information has yet to be elucidated in BED individuals (Davis, 1997).

Lifestyle or unstructured PA has been successfully utilized in other populations to increase long-term adherence to PA and to improve physiological outcomes such as cardiovascular endurance (Dunn, Garcia, Marcus, Kampert, Kohl, \& Blair, 1998) (Dunn, Marcus, Kampert, Garcia, Kohl, \& Blair, 1999). The addition of unstructured PA may be an important addition to treatment or a protective factor from excess body weight commonly associated with BED. A paucity of information describes the historical and current PA participation of BED individuals.

Given the need for additional information on individuals with BED, the primary aim of this study was to better understand how PA involvement changes throughout the life spans of BED participants, subclinical BED participants, and non-BED overweight controls. A secondary aim was to determine if perceived barriers exist that might limit PA participation in these groups. The findings from this study may aid in the promotion of structured and unstructured PA as an adjunct treatment for BED.

\section{Methods}

\section{Participants}

Phase I: Females $(N=312)$ between the ages of 18 and 50 were recruited from communities within the continental United States with the goal of obtaining a convenience sample of 75 participants who met all five criteria for BED. Recruited from multiple sources, including religious organizations, educational groups, and eating disorder treatment groups, all participants were self-administered a series of questionnaires. A cover letter accompanied the questionnaires and informed participants that completion of the questionnaires indicated their consent to participate in the study. The questionnaires consisted of a (a) Demographic Questionnaire; the (b) Questionnaire on Eating and Weight Patterns-Revised; the (c) Exercise Benefits To Barriers Scale (EBBS); and the (d) Bone Loading History Questionnaire (Yanovski, Nelson, Dubbert, \& Spitzer, 1993) (Kriska, Sandler, Cauley, Laporte, Hom, \& Pambianco 1988) (Dolan, Williams, Ainsworth, \& Shaw, 2006). Following is a more detailed description of each questionnaire.

\section{Questionnaire on Eating and Weight}

\section{Patterns-Revised}

The Questionnaire on Eating and Weight Patterns-Revised (QEWP) was chosen to screen participants for binge eating status. The QEWP, a four-page questionnaire that contains 28 items, was developed to diagnose BED using DSM-IV-TR criteria (Yanovski, 1993).Diagnoses are based only on the responses to 13 out of a possible 28 items. These 13 items classify individuals into one of four categories: (a) $\mathrm{BED}$, (b) purging bulimia nervosa, (c) nonpurging bulimia 
nervosa, or (d) not classified as a binge eater. Reported internal consistency was .75 in a weight control sample and .79 in a community sample (Morgan et al., 2002).The QEWP derived diagnosis of BED has also been reported to agree with the structured interview diagnosis (kappa $=.57$ ) (Johnson \& Torgrud, 1996) (Pratt, Telche, Labouvie, Wilson, \& Agras, 2001). The QEWP contains a question that ascertains binge eating severity or the average number of binge eating episodes per week in the last 6 months. Possible responses are $1=$ less than 1 day per week, $2=1$ day per week, $3=2$ or 3 days per week, or $4=4$ or 5 days per week or nearly every day (Yanovski, 1993). Participants self-reported body weight and height for the calculation of body mass index.

\section{Exercise Benefits To Barriers Scale}

The Exercise Benefits to Barriers Scale (EBBS) was used to determine the participants' perceived benefits and barriers to exercise (Sechrist, Walker, \& Pendler, 1987). Containing 40 items, the EBBS uses a 4-point, forced-choice, Likert-type format to obtain an ordinal measure of the strength of agreement with item statements. Benefits were scored as $4=$ strongly agree, $3=$ agree, $2=$ disagree, and $1=$ strongly disagree. Barriers were reverse scored. Two scores were calculated: (a) total benefits $\left(\mathrm{EBBS}_{\mathrm{ben}}\right)$ and (b) total barriers $\left(\mathrm{EBBS}_{\mathrm{bar}}\right)$. The possible range of scores on the EBBS $_{\text {ben }}$ is between 29 and 116, with most scores falling between 51 and 116. On the $\mathrm{EBBS}_{\mathrm{bar}}$ the possible range of scores is between 14 and 56, with most scores falling between 14 and 56. The standardized Cronbach's alpha reliability coefficients were .953 for the $\mathrm{EBBS}_{\text {ben }}$ and .886 for the $\mathrm{EBBS}_{\text {bar }}$ (Vuillenium et al., 1987).

\section{Bone Loading Health Questionnaire}

The Bone Loading Health Questionnaire (BLHQ) was designed to ascertain historical PA specific to bone (Cronbach's alpha was .86) (Dolan et al., 2006).To measure both historical and current PA in the participants, the BLHQ was modified so that frequency, intensity, and duration could be recorded for the specific PAs performed during the identified time periods: (a) elementary school, (b) junior high school, (c) high school, (d) young adult (18 to 29 years old), and (e) mature adult (30 to 50 years old). For the school-age time periods, PA during school hours and leisure time PA were ascertained. Leisure time PA was determined for the young adult and mature adult time periods. Participants were provided with a sample list of PAs. These activities included structured activities (e.g., basketball and volleyball) and lifestyle or unstructured activities (e.g., gardening and hiking). Occupational PA was not measured with the BLHQ. Two outcome measures for PA were calculated: (a) mean hours per week $\left(\mathrm{h} \cdot \mathrm{wk}^{-1}\right)$ and $(\mathrm{b})$ mean metabolic equivalent unit (MET) hours per week (MET-h $\left.\cdot \mathrm{wk}^{-1}\right)$.

Detailed information was collected for frequency and duration of each PA. Participants indicated the number of months per year of participation and the number of years of participation during each time period. Mean hours per week of PA were calculated by summing the hours of participation for all activities (see Equation 1) (Pendleton et al., 2000). The estimated metabolic cost of PA participation during each time period was obtained by multiplying the number of hours by its estimated metabolic cost (MET) (see Equation 2).

(yr participated in activity) x (mo/yr) x $(4 \mathrm{wk} / \mathrm{mo}) \mathrm{x}(\mathrm{h} / \mathrm{wk})$

$\div$ (total $\mathrm{yr}$ in period $) \div(52 \mathrm{wk} / \mathrm{yr})=\mathrm{h} \cdot \mathrm{wk}^{-1}$

$\mathrm{h} \cdot \mathrm{wk}^{-1} \mathrm{x}$ mean MET value $=$ MET-h $\cdot \mathrm{wk}^{-1}$

MET values represent a general estimate of the intensity of an activity. One MET equals an oxygen utilization of 3.5 $\mathrm{ml} / \mathrm{kg} / \mathrm{min}$ or roughly $1 \mathrm{kcal} / \mathrm{kg} / \mathrm{min}$ for an individual with a resting metabolic rate of $60 \mathrm{kcal} / \mathrm{hr}$ (e.g., body mass $\cong 60 \mathrm{~kg}$ ) (Ainsworth et al., 2000). MET intensities were categorized as light (1.5 METs), moderate (4.0 METs), vigorous (6.0 METs), and very vigorous (8.0 METs) (Matthews et al., 2000). The most common PAs for each time period for the BED participants were also determined using the Bone Loading Health Questionnaire.

Phase II: After surveying the initial sample of 312 females, 19 participants met the BED criteria representing a prevalence rate of $6 \%$ (See Table 1). Two control groups were identified from the initial sample. First, participants who met at least two of the diagnostic criteria for BED were assigned to a subclinical BED group $(n=18)$. Second, a body weight matched sample of the remaining participants who did not meet any of the diagnostic criteria (non-BED) $(n=19)$ was also selected to serve as controls.

\section{Statistical Analysis}

Multiple one-way analysis of variance (ANOVA) was used to compare age, body mass index, body weight, highest body weight, exercise benefits to barriers scores, and PA levels $\left(\mathrm{h} \cdot \mathrm{wk}^{-1}\right.$; MET- $\left.\mathrm{h} \cdot \mathrm{wk}^{-1}\right)$ among groups. PA variables that did not meet the homogeneity of variance assumption were $\log$ transformed before analysis and transformed back into original units to facilitate data interpretation. Multiple one-way ANOVA was used to compare $\mathrm{EBBS}_{\text {ben }}$ and EBBS $_{\mathrm{bar}}$ among the three groups. Bonferroni corrections were made to control for Type I error rate inflation. Pearson chi-square analyses were used to compare individual benefits and barriers questions among groups and to determine if the groups differed on education level and ethnicity. Statistical significance was accepted at an alpha level of $p<0.05$ except where Bonferoni corrected and noted.

\section{Results}

All analyses were performed using SPSS. Multiple one-way ANOVAs were used to compare age, body mass index, current body weight, highest body weight, exercise benefits to barriers scale scores, and PA levels $\left(\mathrm{h} \cdot \mathrm{wk}^{-1}\right.$; MET-h $\left.\cdot w^{-1}\right)$ among groups. No significant differences were found in body mass index, current body weight, highest body weight, exercise benefits to barriers scale scores, or MET-h $\cdot \mathrm{wk}^{-1}$ (see Table 1). Significant differences were found among groups in $\mathrm{h} \cdot \mathrm{wk}^{-1}$ for the high school, young adult, and mature adult time periods (see Table 2). Table 3 presents the most common PAs for the BED group during 
each time period.

Table 1. Participant Characteristics.

\begin{tabular}{cccc}
\hline $\begin{array}{c}\text { Initial charac- } \\
\text { teristics }\end{array}$ & $\begin{array}{c}\text { BED } \\
(n=19)\end{array}$ & $\begin{array}{c}\text { Subclinical } \\
\text { BED } \\
(n=18)\end{array}$ & $\begin{array}{c}\text { Controls } \\
(n=19)\end{array}$ \\
\hline Age & 38.26 & 35.78 & 34.32 \\
(year; $M \pm S D)$ & $(15.81)$ & $(13.62)$ & $(3.05)$ \\
Have a college & 26 & 42 & 37 \\
degree (\%) & & & \\
Caucasian (\%) & 100 & 95 & 89 \\
Body mass & 31.76 & 29.52 & 31.84 \\
index $\left(\mathrm{kg} / \mathrm{m}^{2} ;\right.$ & $(7.44)$ & $(6.56)$ & $(6.84)$ \\
$M \pm S D)$ & & & \\
Body weight & 191.53 & 181.48 & 192.25 \\
(kgs; $M \pm S D)$ & $(45.11)$ & $(42.28)$ & $(45.24)$ \\
Highest body & 202.95 & 196.17 & 198.41 \\
weight $(\mathrm{kgs} ; M$ & $(50.85)$ & $(51.18)$ & $(48.12)$ \\
$\pm S D)$ & & & \\
\hline
\end{tabular}

Note. Education and ethnicity were compared using chi-square. Age, body weight, and highest body weight were compared using ANOVA. $p=.05$.

Table 2. Analysis of Variance for Mean Weekly Physical Activity Levels.

\begin{tabular}{cccc}
\hline BED status & \multicolumn{1}{c}{$\begin{array}{c}p \\
\text { value }\end{array}$} & \multicolumn{1}{c}{$\eta^{2}$} \\
\hline & \multicolumn{1}{c}{ Mean MET hours/week } & \\
Elementary school & .692 & .509 & .049 \\
Junior high school & .517 & .602 & .037 \\
High school & 2.30 & .120 & .145 \\
Young adult & 1.49 & .244 & .099 \\
Mature adult & 2.67 & .088 & .165 \\
& Mean hours/week & \\
Elementary school & .515 & .603 & .037 \\
Junior high school & 1.14 & .336 & .078 \\
High school & 3.67 & $.039^{*}$ & .214 \\
Young adult & 8.66 & $.001^{*}$ & .391 \\
Mature adult & 21.87 & $.000^{*}$ & .618 \\
\hline
\end{tabular}

Note. $\eta^{2}:$ small $=.01$, medium $=.06$, and large $=.14 .{ }^{*} p<.05$.

The overall $F$ tests were significant for $\mathrm{h} \cdot \mathrm{wk}^{-1}$ of PA during the high school, young adult, and mature adult time periods; therefore, follow-up tests were conducted to evaluate pair wise differences among the means. PA data were log transformed prior to analysis. The decision was made to use a post hoc procedure that assumes equal variances (Tukey honestly significant difference test). For the high school time period, the subclinical BED group reported significantly lower PA when compared to the non-BED controls ( $p$ $<.048)$. For the young adult time period, the subclinical BED group had significantly lower PA when compared to the non-BED controls $(p<.004)$, and the BED group had significantly lower PA when compared to the non-BED controls $(p<.003)$. The same trend continued for the mature adult time period, with the BED group reporting significantly lower PA when compared to the non-BED controls, $p<.000$. The subclinical BED group reported significantly lower PA when compared to the non-BED controls, $p<.000$ ).

Multiple one-way ANOVAs were used to compare $E_{\text {BBS }}$ and $\mathrm{EBBS}_{\mathrm{bar}}$ among the three groups. No significant differences were found among these scores. Chi-square analyses were conducted to determine if significant differences existed in individual perceived barriers among the three groups. Table 4 presents seven perceived barriers that were significantly different among groups: (a) exercise costs too much, (b) exercise takes too much time, (c) exercise takes too much time away from family, (d) exercise facility schedules are inconvenient, (e) family members do not encourage exercise, (f) people look funny in exercise clothes, and (g) too few places to exercise. Follow-up pairwise comparisons were conducted to evaluate differences among the three groups. The strength of the relationship among the groups and barriers was assessed using the Cramer's V statistic. Tables 5, 6, and 7 show the results of these pairwise comparisons.

Table 3. Most Common Physical Activities Per Time Period for Binge Eating Disorder Participants.

\begin{tabular}{ccccc}
\hline Rank & Elementary school & Junior high school & High school & Young adult \\
\hline 1 & Children's games & Basketball & Jogging & Aerobic dancing \\
2 & Bicycling & Softball & Walking & Softball, dancing \\
3 & Kickball & Children's games & Swimming,running & Jogging \\
4 & Softball & Bicycling, swimming, & Dancing, exercise, & Swimming \\
\hline
\end{tabular}

Note. Only the top four PAs are listed for each time period. Multiple activities per cell represent similar frequencies. Multiple activities are listed one time only.

Table 4. Comparison of Statistically Significant Perceived Barriers Among Groups.

\begin{tabular}{cccc}
\hline Perceived barrier & $\begin{array}{c}\text { BED } \\
(\mathrm{n}=19 ; \mathrm{M} \pm \mathrm{SD})\end{array}$ & $\begin{array}{c}\text { Subclinical BED } \\
(\mathrm{n}=18 ; \mathrm{M} \pm \mathrm{SD})\end{array}$ & $\begin{array}{c}\text { Non-BED } \\
(\mathrm{n}=19 ; \mathrm{M} \pm \mathrm{SD})\end{array}$ \\
\hline Most facility schedules are inconvenient. & $2.57 \pm .84 * \mathrm{a}$ & $2.84 \pm .37 \mathrm{~b}$ & $3.09 \pm 1.00$ \\
People in exercise clothes look funny. & $2.48 \pm .95 * \mathrm{a}$ & $2.89 \pm .46 \mathrm{~b}$ & $3.22 \pm .85$ \\
Exercise takes too much time & $2.40 \pm .84 \mathrm{a}$ & $2.79 \pm .63$ & $3.13 \pm .81$ \\
$\quad$ away from family & $2.83 \pm 1.08 * \mathrm{a}$ & $3.07 \pm .62$ & $3.04 \pm 1.02$ \\
It costs too much to exercise & $2.83 \pm .98 *$ & $2.89 \pm .57 \mathrm{~b}$ & $3.26 \pm .96$ \\
There are too few places to exercise. & $2.70 \pm .76 \mathrm{a}$ & $2.73 \pm .56$ & $3.00 \pm .85$ \\
\hline My family does not encourage me to exercise & &
\end{tabular}

Note. Responses were made on a 4-point Likert scale $(1=$ strongly agree, $2=$ agree, $3=$ disagree, and $4=$ strongly disagree).

*Significantly different than subclinical BED.

${ }^{a}$ Significantly different than non-BED.

${ }^{\mathrm{b}}$ Subclinical BED significantly different than non-BED.

$p<.05$. 
Table 5. Pairwise Comparisons of Perceived Barriers for Binge Eating Disorder and Subclinical Binge Eating Disorder.

\begin{tabular}{ccccc}
\hline Perceived barrier & Value & $d f$ & Asymp. sig. (2-sided) & Cramer's V \\
\hline Most facility schedules are inconvenient. & 12.88 & 3 & .006 & .58 \\
People in exercise clothes look funny. & 10.52 & 3 & .015 & .53 \\
It costs too much to exercise. & 12.37 & 4 & .015 & .58 \\
There are too few places to exercise. & 10.47 & 3 & .015 & .53 \\
\hline
\end{tabular}
$p<.05$.

Table 6. Pairwise Comparisons of Perceived Barriers for Binge Eating Disorder and Nonbinge Eating Disorder.

\begin{tabular}{ccccc}
\hline Perceived barrier & Value & $d f$ & Asymp. sig. (2-sided) & Cramer's V \\
\hline Most facility schedules are inconvenient. & 12.48 & 3 & .006 & .57 \\
$\begin{array}{c}\text { There are too few places to exercise. } \\
\begin{array}{c}\text { Exercise takes too much time away from } \\
\text { family responsibilities. }\end{array}\end{array}$ & 8.10 & 3 & .044 & .46 \\
$\begin{array}{c}\text { My family does not encourage me to } \\
\text { exercise. }\end{array}$ & 11.27 & 3 & .010 & .54 \\
\hline
\end{tabular}

$p<.05$.

Table 7. Pairwise Comparisons of Perceived Barriers for Subclinical Binge Eating Disorder and Nonbinge Eating Disorder.

\begin{tabular}{ccccc}
\hline Perceived barrier & Value & $d f$ & Asymp. sig. (2-sided) & Cramer's V \\
\hline Most facility schedules are inconvenient. & 11.28 & 3 & .009 & .58 \\
There are too few places to exercise. & 12.17 & 3 & .007 & .57 \\
People in exercise clothes look funny. & 8.26 & 3 & .041 & .47 \\
\hline
\end{tabular}

$\alpha=.05$.

\section{Discussion}

The primary aim of this study was to better understand how PA involvement changes throughout the life spans of BED individuals. The findings revealed that during elementary school and junior high school, BED individuals did not differ from the subclinical or non-BED overweight controls. Statistically significant differences did not exist for high school PA among BED individuals and non-BED overweight controls. However, the BED individuals reported 2.2 and 3.9 more hours of PA per week as compared to non-BED overweight controls and subclinical BED participants, respectively. In addition, the BED individuals and the subclinical BED individuals reported beginning to binge eat in high school at the mean age of $18 \mathrm{y}$, whereas the overweight controls did not report a binge episode until young adulthood at the age of $23 \mathrm{y}$. During junior high school and high school, adolescents, particularly females, begin to compare their bodies to others (Ransdell, Wells, Manore, Swan, \& Corbin, 1998). The possibility exists that BED individuals may have used PA during high school to compensate for the binge eating episodes initiated during high school. BED individuals have been known to infrequently compensate for binge episodes, exhibiting symptoms of nonpurging bulimia nervosa. Determining if PA is used during high school to compensate for binge eating should be an objective for future research.

A related finding was the significantly lower PA levels reported during the young and mature adult periods for BED individuals as compared to non-BED controls. During high school, opportunities for PA within the school setting are more available and family and work responsibilities are relatively few. Researchers have shown that family and work responsibilities limit PA participation thus, the combination of more PA opportunities and fewer responsibilities allows for individuals to be more physically active during high school (Sherwood \& Jeffery, 2000). All of the groups examined in this study were more active in high school as compared to the young and mature adult periods. Therefore, it appears BED participants were physically active as children and became less active as young adults and mature adults without concurrent reductions in caloric intake. Increases in body weight later in life are likely due to these lifestyle changes, therefore future research is needed to determine if PA may affect early binge eating and the weight consequences of BED.

Other important questions can be addressed using the PA data of BED individuals from this study. First, it is important to determine if BED participants were physically active in elementary school, junior high school, and high school in sufficient amounts to produce significant health benefits. Second, it is important to determine if BED participants are receiving sufficient amounts of $\mathrm{PA}$ for weight management as adults. Finally, comparisons of BED participants' PAs with data from other populations can provide a more descriptive picture of their PA patterns.

The BED participants in this study reported an average of 55.37 minutes of PA per day during elementary school and an average of 51.60 and 71.57 minutes per day during junior high school and high school, respectively. The Centers for Disease Control recommends that elementary school students receive a minimum of 60 minutes a day of moderate and vigorous PA, whereas junior high school and high school students should accumulate a minimum of 30 minutes a day (CDC, 2007). From the current study, it appears that BED participants received more than sufficient amounts of PA during their school-age years to produce health benefits, but 
future research, including objective measurement of PA in this population, is needed to determine if BED individuals use PA to compensate for binge eating episodes.

The BED participants in this study participated in an average of $2.25 \mathrm{~h} \cdot \mathrm{wk}^{-1}$ of PA at an average intensity of 4.9 METs and $1.1 \mathrm{~h} \cdot \mathrm{wk}^{-1}$ at an average intensity of 5.01 METs during their young adult and mature adult years, respectively. Donnelly and colleagues (2009) recommended that overweight and obese individuals progressively increase PA from $3.3 \mathrm{~h} \cdot \mathrm{wk}^{-1}$ to $5 \mathrm{~h} \cdot \mathrm{wk}^{-1}$. Increasing PA participation to greater than $5 \mathrm{~h} \cdot \mathrm{wk}^{-1}$ may facilitate improved long-term maintenance of weight loss. ${ }^{7}$ From these results, it appears that BED participants participated in sufficient amounts of PA for reductions in chronic disease risks during their young adult years, but did not participate in recommended amounts to facilitate long-term weight maintenance. PA participation during their mature adult years was almost $6 \mathrm{~h} \cdot \mathrm{wk}^{-1}$ less than recommended by the American College of Sports Medicine for weight maintenance.

The reductions in PA and intensity over the life span found in this study are similar to those found in other studies (CDC, 2007).It is difficult to ascertain from the present study the reasons for the reductions in PA throughout the life span; however, the barriers found from administration of the EBBS provide evidence that specific barriers may affect PA levels of BED individuals. A closer examination of the types of PAs participated in by the BED participants across the life span may help to clarify issues related to reduced PA and intensity.

As participants grew older, their PAs became less structured. Expectedly, children's games were the predominate activities listed during elementary school age, whereas structured activities such as basketball and softball were popular during junior high school age. As the participants moved into high school, their activities became less team or sport oriented and more fitness oriented (e.g., jogging and walking were the most popular reported activities). As young adults (18 to 29 years old), aerobic dance and softball were popular. Mature adults (30 to 50 years old) participated in more unstructured or lifestyle activities such as walking, hiking, and gardening. Because the participants reported a greater number of unstructured or lifestyle activities, it may be helpful to prescribe these types of PAs during BED treatment.

The high body mass indices, body weights, and highest body weights reported for the BED participants are in agreement with values reported by other researchers (Dingemans et al., 2002). These results are not surprising because individuals meeting the diagnostic criteria for BED report high caloric intakes as a result of their binge eating episodes (Gueritn, 1999).The World Health Organization classification scheme defines overweight as a body mass index between 25 and 29.9, whereas obesity is set at a body mass index of 30 and above (Westerterp, 1999). When the BED participants for this study were compared to these standards, $17 \%$ of the sample was considered overweight and $61 \%$ were obese.
No significant differences were found in perceived benefits to barriers scores among any of the groups. Ransdell and colleagues (1998) examined changes in perceived benefits and barriers as a result of participation in home-based and university-based PA interventions. Prior to the intervention, the baseline-perceived benefits to barriers scores were similar to the participants in this study, suggesting that significant and practical differences do not exist between BED participants and other populations when composite scores for perceived benefits and barriers are used. However, there are significant differences when specific perceived barriers are examined separately.

Specific perceived barriers were identified by BED participants that were significantly different from subclinical BED participants and controls. BED participants believed that (a) exercise costs too much, (b) exercise takes too much time, (c) exercise takes too much time away from family, (d) exercise facility schedules inconvenient, (e) family members do not encourage exercise, (f) people look funny in exercise clothes, and (g) too few places to exercise. In a comprehensive review, Sherwood and Jeffery stressed the importance of addressing prominent barriers and enhancing social support to PA to ensure long-term adherence (Sherwood, 2000). Because these barriers have not been described in the BED population, they can now be addressed during the design and implementation of PA interventions for BED participants to improve both the initiation of PA and adherence to programs.

An important overall finding from the present study is that there were differences in specific perceived barriers to exercise between the BED participants and the subclinical BED participants. Crow and colleagues questioned whether or not the diagnostic criteria for BED make meaningful distinctions between full and partial (subclinical) cases of BED (Fitzgibbon \& Blackman, 2000). They found that $30 \%$ of bulimia nervosa cases were incorrectly classified as BED, and $17 \%$ of BED cases were incorrectly classified as bulimia nervosa. Others have concluded that the current diagnostic criteria are sufficient to delineate between BED and bulimia nervosa (Crow, Agras, Halmi, Mitchell, \& Kraemer, 2002) (Streigel-Moore et al., 2001). The results of this study provide evidence that the diagnostic criteria do delineate between BED and partial BED. Because the two groups differed in perceived barriers to exercise in this study, this variable may be a useful addition for the identification of BED.

The descriptions of PA levels, intensities, perceived benefits of and barriers to PA, and common PAs across the life span are informative because there is no previous research describing these variables in BED participants. The PA levels reported by BED participants in this study were not sufficient to produce significant weight loss or to reduce the risk of weight gain over time (Donnelley et al., 2009).

The results must be interpreted with caution due to specific limitations associated with the exploratory nature of this study. First, difficulty in recruiting BED participants resulted in a lower than expected sample size. Second, a newly developed mechanical bone loading questionnaire was used to 
measure energy expenditure. An existing questionnaire with established validity and reliability may have been a better choice for the measurement of energy expenditure, particularly with overweight or obese individuals.

Factors related to PA program adherence were a major focus of this investigation. Specific perceived barriers to PA were identified in BED participants, yet many questions remain related to these barriers. Further exploring the PA perceptions of BED participants would be an important next step in answering these questions. Determining how the participants defined PA, their attitudes about PA, and their eating patterns related to PA could aid in optimal PA programming. Finally, because the tool contained a limited number of possible barriers that the participants could choose from in the EBBS, there may be other perceived barriers yet to be identified. Modification of the EBBS to include these potential barriers may provide information that could be used to design a more optimal PA intervention. In combination, all of this information could be used to design PA interventions for BED participants that incorporate the latest in behavioral research (Ransdell et al., 1998). Future research should attempt to apply qualitative research methodologies to enrich one's understanding of the quantitative data from this study. The following questions need to be answered. How do BED participants define PA? What are BED participants' attitudes about PA? How are BED participants' eating patterns related to PA? What effect could these eating patterns have on optimal PA programming?

\section{Conclusions}

The objective of this study was to better describe how PA involvement changes in BED individuals throughout their life span. In addition, BED participants' perceived benefits of and barriers to PA were assessed to determine if barriers existed that might limit PA participation.

BED participants did not significantly differ in body mass indices, current body weights, or highest body weights. No significant differences were found in mean MET hours per week; however, significant differences were found in mean hours of PA per week. Significant differences were found in perceived barriers to exercise between BED participants and controls.

It can be concluded that BED participants are physically active as children and adolescents. The BED participants reported beginning to binge eat in high school around the age of $18 \mathrm{y}$. BED participants participated in sufficient amounts of PA in high school, but the possibility does exist that PA was used to compensate for binge eating episodes. Large reductions in PA were found after the participants left high school. Although not completely sedentary, they did not participate in sufficient amounts of PA to produce significant health benefits or to manage body weight. The reductions in PA may be explained by the specific perceived barriers identified by the BED participants

\section{REFERENCES}

[1] Agras, S. W. (1995). Treatment of the obese binge eater. In K. D. Brownell \& C. G. Fairburn (Eds.), Eating disorders and obesity (p. 538). New York: The Guilford Press

[2] Ainsworth, B., Haskell, W., Whitt, M., Irwin, M., Swartz, A., Strath, S., O'Brien, W., Bassett, D., Schmitz, K., Emplaincourt, P., Jacobs, D., \& Leon, A. (2000). Compendium of physical activities: An update of activity codes and MET intensities. Medicine and Science in Sports and Exercise, 32, S498-S516

[3] Brownley, K.A., Berkman, N.D., Sedway, J.A., Lohr, K.N., \& Bulik, C.M. (2007). Binge Eating Disorder: A systematic review of randomized controlled trials. International Journal of Eating Disorders, 40, 337-348

[4] Centers for Disease Control (2007).Prevalence of regular physical activity among adults - United States, 2001 and 2005. Morbidity and Mortality Weekly Report, 56, 1209-1212

[5] Crandall, K. J., \& Eisenman, P. A. (2001). Physical activity: A treatment option for binge eating disorder? Women in Sport and Physical Activity Journal, 10, 95-116

[6] Crow, S. J., Agras, W. S., Halmi, K., Mitchell, J. E., \& Kraemer, H. C. (2002). Full syndromal versus subthreshold anorexia nervosa, bulimia nervosa, and binge eating disorder: A multicenter study. New York: Wiley Periodicals

[7] Davis, C., Katzman, D. K., Kaptein, S., Kirsh, C., Brewer, H., Kalmbach, K., Olmsted, M. P., Woodside, D. B., \& Kaplan, A. S. (1997). The prevalence of high-level exercise in the eating disorders: Etiological implications. Comprehensive Psychiatry, 38(6), 321-326

[8] de Zwaan, M., Aslam, Z., \& Mitchell, J. E. (2002). Research on energy expenditure in individuals with eating disorders: A review. International Journal of Eating Disorders, 31, 361-369

[9] Dingemans, A. E., Bruna, M. J., \& Furth, E. F. (2002). Binge eating disorder: A review. International Journal of Obesity, 26, 299-307

[10] Dolan, Shawn H., Williams, Daniel P., Ainsworth, Barbara E. and Shaw, Janet M. (2006-06) Development and Reproducibility of the Bone Loading History Questionnaire. Medicine and Science in Sports and Exercise, 38 (6), 1121-1131

[11] Donnelly, J.E., Blair, S.N., Jakicic, J.M., Manore, M.M. Rankin, J.J., Smith, B.K. (2009). Appropriate Physical Activity Intervention Strategies for Weight Loss and Prevention of Weight Regain for Adults. Medicine \& Science in Sports \& Exercise, 41(2), 459-471

[12] Dunn, A. L., Garcia, M. E., Marcus, B. H., Kampert, J. B., Kohl, H. W., \& Blair, S. N. (1998). Six-month physical activity and fitness changes in Project Active, a randomized trial. Medicine and Science in Sports and Exercise, 30(7), 1076-1083

[13] Dunn, A. L., Marcus, B. H., Kampert, J. B., Garcia, M. E., Kohl, H. W., \& Blair, S. N. (1999). Comparison of lifestyle and structured interventions to increase physical activity and cardiorespiratory fitness. Journal of the American Medical Association, 281(4), 327-334 
[14] Fitzgibbon, M. L., \& Blackman, L. R. (2000). Binge eating disorder and bulimia nervosa: Differences in the quality and quantity of binge eating episodes. International Journal of Eating Disorders, 27, 238-243

[15] Grilo, C.M., White, M.A., \& Masheb, R.E. (2009). DSM-IV Psychiatric comorbidity and its correlates in binge eating disorder. International Journal of Eating Disorders, 42, 228-234

[16] Guertin, T. L. (1999). Eating behavior of bulimics, self-identified binge eaters, and non-eating-disordered individuals: What differentiates these populations? Clinical Psychology Reviews, 19(1), 1-23

[17] Johnson, W.G., \& Torgrud, L.J. (1996). Assessment and treatment of binge eating disorder. In J.K. Thompson (Ed.), Body image: Eating disorders and obesity (pp. 321-343). Washington, DC: American Psychologic

[18] Kriska, A. M., Sandler, R. B., Cauley, J. A., Laporte, R. E., Hom, D. L., \& Pambianco, G. (1988). The assessment of historical physical activity and its relation to adult bone parameters. American Journal of Epidemiology, 127, $1053-1063$

[19] Levine, M. D., Marcus, M. D., \& Moulton, P. (1996). Exercise in the treatment of binge eating disorder. International Journal of Eating Disorders, 19(2), 171-177

[20] Levine, M.D. \& Marcus, M.D. (2003). Psychosocial treatment of binge eating disorder: An update. Eating Disorders Review, 14, 3

[21] Matthews, C. E., Freedson, P. S., Herbert, J. R., Stanek, E. J., Merriam, P. A., \& Ockene, I. S. (2000). Comparing physical activity assessment methods in the Seasonal Variation of Blood Cholesterol Study. Medicine and Science in Sports and Exercise, 32, 976-984

[22] Morgan, C. M., Yanovski, S. Z., Nguyen, T. T., McDuffie, J., Sebring, N. G., Jorge, M. R., Keil, M., \& Yanovski, J. A. (2002). Loss of control over eating, adiposity, and psychopathology in overweight children. International Journal of Eating Disorders, 31, 430-441

[23] Pendleton, V. R., Goodrick, G. K., Poston, W. S. C., Reeves, R. S., \& Foreyt, J. P. (2002). Exercise augments the effects of cognitive-behavioral therapy in the treatment of binge eating disorder. International Journal of Eating Disorders, 31, $172-184$
[24] Pratt, E. M., Telch, C. F., Labouvie, E. W., Wilson, G. T., \& Agras, W. S. (2001). Perfectionism in women with binge eating disorder. International Journal of Eating Disorders, 29, 177-186

[25] Ransdell, L. B., Wells, C. L., Manore, M. M., Swan, P. D., \& Corbin, C. B. (1998). Social physique anxiety in postmenopausal women. Journal of Women and Aging, 10(3), 19-39

[26] Sechrist, K. R., Walker, S. N., \& Pender, N. J. (1987). Development and psychometric evaluation of the exercise benefits/barriers scale. Research in Nursing and Health, 10, 357-365

[27] Sherwood, N. E., \& Jeffery, R. W. (2000). The behavioral determinants of exercise: Implications for physical activity interventions. Annual Review of Nutrition, 20, 21-44

[28] Spitzer, R. L., Yanovski, S., Wadden, T., Wing, R., Marcus, M. D., Stunkard, A., Devlin, M., Mitchell, J., Hasin, D., \& Horne, R. L. (1993). Binge eating disorder: Its further validation in a multisite study. International Journal of Eating Disorders, 13(2), 137-153

[29] Striegel-Moore, R. H., Cachelin, F. M., Dohm, F., Pike, K. M., Wilfley, D. E., \& Fairburn, C. G. (2001). Comparison of binge eating disorder and bulimia nervosa in a community sample. International Journal of Eating Disorders, 29, $157-165$

[30] Vuillemin, A., Oppert, J. M., Guillemin, F., Essermeant, L., Fontvieille, A. M., Galan, P., Kriska, A. M., \& Hercberg, S. (2000). Self-administered questionnaire compared with interview to assess past-year physical activity. Medicine and Science in Sports and Exercise, 32, 1119-1124

[31] Westerterp, K. R. (1999). Obesity and physical activity. International Journal of Obesity and Related Metabolic Disorders, 23(Suppl. 1), S59-S64

[32] Wonderlich, S.A., Gordon, K.H., Mitchell, J.E., Crosby, R.D., \& Engel, S.G. (2009). The validity and clinical utility of binge eating disorder. International Journal of Eating Disorders, 42, $687-705$

[33] Yanovski, S. (1993). Binge eating disorder: Current knowledge and future directions. Obesity Research, 1, 306-324

[34] Yanovski, S. Z., Nelson, J. E., Dubbert, B. K., \& Spitzer, R. L. (1993). Association of binge eating disorder and psychiatric comorbidity in obese subjects. American Journal of Psychiatry, 150(10), 1472-1479 